\title{
Post-Treatment Techniques for Enhancing Mode-Coupling in Long Period Fiber Gratings Induced by $\mathrm{CO}_{2}$ Laser
}

\author{
Xizhen XU, Jian TANG, Jing ZHAO, Kaiming YANG, Cailing FU, Qiao WANG, \\ Shen LIU, Changrui LIAO, Jiarong LIAN, and Yiping WANG*
}

Key Laboratory of Optoelectronic Devices and Systems of Ministry of Education and Guangdong Province, Shenzhen University, Shenzhen, 518060, China

"Corresponding author: Yiping WANGＥ-mail: ypwang@szu.edu.cn

\begin{abstract}
Two promising post-treatment techniques, i.e. applying tensile strain and rising temperature, are demonstrated to enhance the mode-coupling efficiency of the $\mathrm{CO}_{2}$-laser-induced long period fiber gratings (LPFGs) with periodic grooves. Such two post-treatment techniques can be used to enhance the resonant attenuation of the grating to achieve a LPFG-based filter with an extremely large attenuation and to tailor the transmission spectrum of the $\mathrm{CO}_{2}$-laser-induced LPFG after grating fabrication.
\end{abstract}

Keywords: Long period fiber gratings, fiber Bragg gratings, optical fiber sensor, temperature, tensile strain, optical fiber device.

Citation: Xizhen XU, Jian TANG, Jing ZHAO, Kaiming YANG, Cailing FU, Qiao WANG, et al., "Post-Treatment Techniques for Enhancing Mode-Coupling in Long Period Fiber Gratings Induced by $\mathrm{CO}_{2}$ Laser," Photonic Sensors, 2015, 5(4): 339-344.

\section{Introduction}

Since Davis et al. reported the first $\mathrm{CO}_{2}$-laserinduced long period fiber grating (LPFG) in a conventional glass fiber in 1998 [1], various $\mathrm{CO}_{2}$ laser irradiation techniques have been demonstrated and improved to write high-quality LPFGs in different types of optical fibers such as conventional glass fibers [2, 3], solid-core photonic crystal fibers $[4,5]$, and air-core photonic bandgap fibers [6]. The enhancement of the grating-writing efficiency is critical to achieving a high-quality LPFG with desired mode-coupling efficiency. A few preprocessing techniques, e.g. hydrogen loading [7] and applying prestrain [2, 8-10], have been demonstrated to enhance the writing efficiency of the $\mathrm{CO}_{2}$-laser-induced LPFGs. As is well known, the use of hydrogen loading can enhance the photosensitivity of the Ge-doped fibers to ultra violet (UV) exposure [11]. Hydrogen loading is also found to enhance the writing efficiency of the $\mathrm{CO}_{2}$-laser- induced LPFGs [1, 7]. Additionally, numerous experiments reveal that the writing efficiency of the $\mathrm{CO}_{2}$-laser-induced LPFGs could be enhanced by means of applying a tensile strain to the fiber employed during $\mathrm{CO}_{2}$ laser irradiation $[2,8,9]$, which may be due to the frozen-in viscoelasticity in the fiber. For example, as shown in Fig. 1 in [2], one of the fiber ends was tensed by a small weight to provide an external tensile strain in the fiber in advance, thus enhancing the efficiency of the grating fabrication [2]. All of these techniques above are

Received: 31 August 2015 / Revised: 10 September 2015

(C) The Author(s) 2015. This article is published with open access at Springerlink.com

DOI: $10.1007 / \mathrm{s} 13320-015-0277-5$

Article type: Regular 
used to enhance mode coupling during the writing process of grating, rather than after grating fabrication. In other words, they are so-called preprocessing techniques [12].

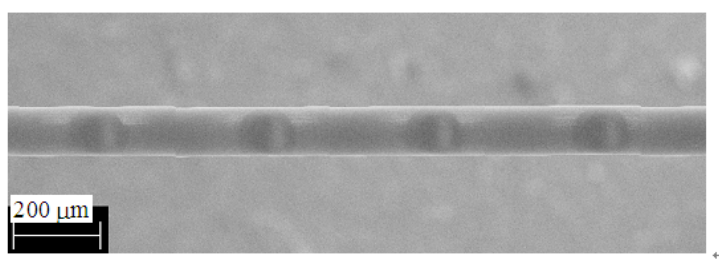

Fig. 1 SEM image of the asymmetric $\mathrm{CO}_{2}$-laser-induced LPFG with periodic grooves (grating pitch: $400 \mathrm{~m}$; number of periods: 20).

Authors recently reported a novel technique for writing an asymmetric LPFG by means of carving periodic grooves on the surface of an optical fiber with a focused $\mathrm{CO}_{2}$ laser beam [13]. Mode coupling in such an asymmetrical $\mathrm{CO}_{2}$-laser-induced LPFG with periodic grooves is very sensitive to the tensile strain applied and the change in external temperature. Based on these unique strain and temperature response of the $\mathrm{CO}_{2}$-laser-induced LPFGs, we demonstrated two promising post-treatment techniques, i.e. applying tensile strain and rising temperature, for enhancing mode-coupling efficiency of the achieved LPFGs. Such post-techniques could be used to tailor the transmission spectrum of LPFGs after grating fabrication.

\section{Applying tensile strain}

An asymmetrical LPFG with 20 periods and a grating pitch of $400 \mathrm{~m}$ were written in a standard singe mode fiber (SMF) by the use of the $\mathrm{CO}_{2}$-laser-carving technique reported in [13]. Periodic grooves were carved on the single-side surface of the fiber, as shown in Fig. 1. The resonant wavelength of this LPFG and the corresponding peak attenuation were $1512.51 \mathrm{~nm}$ and $-28.34 \mathrm{~dB}$, respectively. One end of the LPFG was fixed; whereas another end of the LPFG was tensed by a stretching force to investigate the response of mode coupling in the grating to the tensile strain applied.
As shown in Fig. 2, the resonant wavelength of the $\mathrm{CO}_{2}$-laser-induced LPFG with periodic grooves shifted toward the shorter wavelength with an increase in the tensile strain. And the peak attenuation at the resonant wavelength was enhanced from $-28.34 \mathrm{~dB}$ to $-54.82 \mathrm{~dB}$ while the tensile strain applied was increased to $305 \mu \varepsilon$ and then reduced to $-48.45 \mathrm{~dB}$ while the tensile stain further was increased to $620 \mu \varepsilon$. Moreover, the polarization dependence in the $\mathrm{CO}_{2}$-laser-induced LPFG also was enhanced and then reduced with an increase in the tensile strain applied, as shown in Fig. 3. Maximum polarization dependent loss (PDL) in the grating was enhanced to $27.3 \mathrm{~dB}$ while the tensile strain applied was increased to $305 \mu \varepsilon$.

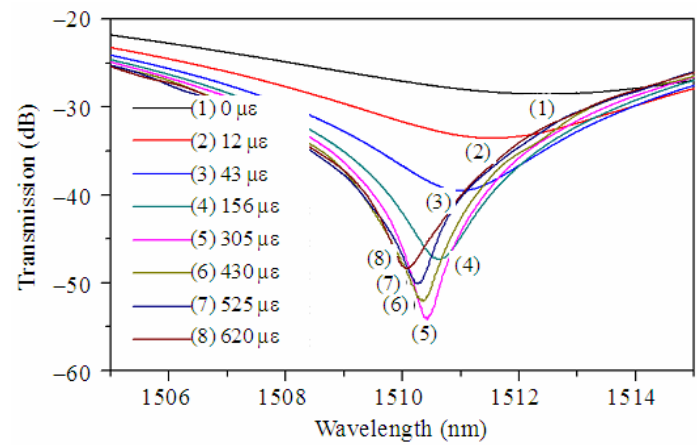

(a)

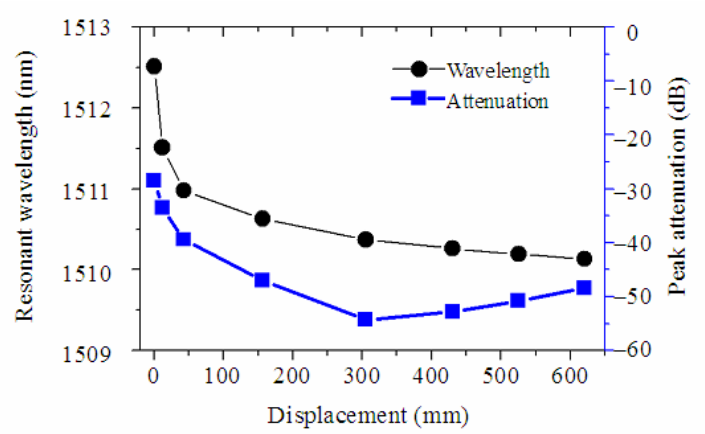

(b)

Fig. 2 Strain response of the $\mathrm{CO}_{2}$-laser-induced LPFG: (a) transmission spectrum evolution of the $\mathrm{CO}_{2}$-laser-induced LPFG with an increase of the tensile stain applied, and (b) resonant wavelength and peak attenuation of the LPFG as a function of the tensile strain.

Such evolutions of the transmission spectrum and polarization dependence in the $\mathrm{CO}_{2}$-laserinduced LPFG are attributed to the enhanced coupling from the fundamental mode to the cladding mode, resulting from the stretch-induced periodic 


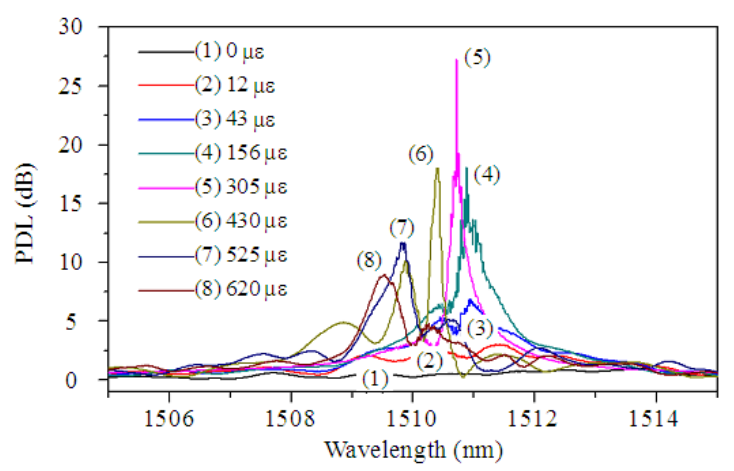

Fig. 3 Polarization dependence evolution of the $\mathrm{CO}_{2}$-laser-induced LPFG with an increase in the tensile stain applied.

microbends of the fiber. As shown in Fig. 4, small lateral bends, i.e. periodic microbends, will be induced, resulting from the asymmetric structure, at each grooved section while the $\mathrm{CO}_{2}$-laser-induced LPFG with asymmetric grooves is stretched longitudinally $[14,15]$. As a result, additional refractive index perturbation is induced by the stretch-induced microbend in the grating due to the photoelastic effect, thus enhancing refractive index modulation in the $\mathrm{CO}_{2}$-laser- induced LPFG, which is similar to the case of the microbend-induced LPFG [16-18]. Consequently, the coupling from the fundamental mode to the cladding modes in the grating is enhanced with an increase in the tensile strain applied, which could be considered as the continuance of the grating writing process. When the tensile strain is increased to a critical value of about $305 \mu \varepsilon$, the deepest attenuation dip of $-54.82 \mathrm{~dB}$ is observed at the resonant wavelength of $1510.40 \mathrm{~nm}$, indicating that the light at the resonant wavelength is almost fully coupled into the cladding mode. In other words, the optimum coupling occurs in the grating because the exact coupling condition of $k L=\pi / 2$ is achieved, where $k$ is the coupling coefficient and $L$ is the grating length. Then the so-call over-coupling occurs with a further increase in the tensile strain. In other word, the light that has been coupled into the cladding mode starts to couple back into the core mode. Thus the peak attenuation is gradually reduced while a stronger tensile strain of more than $305 \mu \varepsilon$ is applied. Since the microbend-induced refractive index perturbation in the grating is asymmetric within the cross-section of the fiber, the polarization dependence also is enhanced with an increase in the tensile strain applied, as shown in Fig. 3.

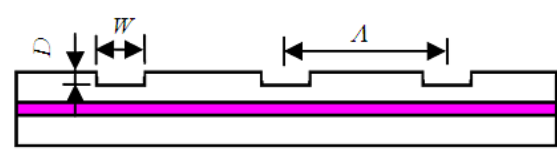

(a)

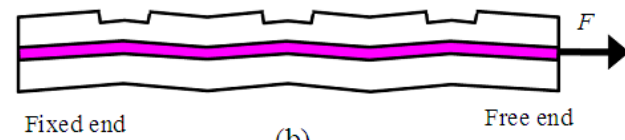

(b)

Fig. 4 Schematic diagram of the $\mathrm{CO}_{2}$-laser-induced LPFG with asymmetric grooves (a) before and (b) after a stretching force is applied to the grating.

\section{Rising temperature}

To demonstrate another post-treatment technique for enhancing the mode coupling in the grating, we wrote another asymmetrical LPFG with 20 periods and grating pitch of $405 \mu \mathrm{m}$ in a standard SMF by the use of the same technique. The resonant wavelength of this $\mathrm{CO}_{2}$-laser-induced LPFG with periodic grooves and the corresponding peak attenuation were $1516.90 \mathrm{~nm}$ and $-39.34 \mathrm{~dB}$, respectively. Then we measured the response of the $\mathrm{CO}_{2}$-laser-induced LPFG to the change in external temperature. As shown in Fig. 5, the resonant wavelength of the $\mathrm{CO}_{2}$-laser-induced LPFG with periodic grooves shifted from $1516.90 \mathrm{~nm}$ toward the longer wavelength of $1522.89 \mathrm{~nm}$ while the temperature rose from $20^{\circ} \mathrm{C}$ to $100^{\circ} \mathrm{C}$. And the peak attenuation at the resonant wavelength was enhanced from $-39.34 \mathrm{~dB}$ to $-48.53 \mathrm{~dB}$ with the temperature rising. Moreover, the polarization dependence was also enhanced with the rise of the temperature, as shown in Fig. 6. The maximum PDL in the grating was enhanced to $6.83 \mathrm{~dB}$ when the temperature rose to $100^{\circ} \mathrm{C}$. 


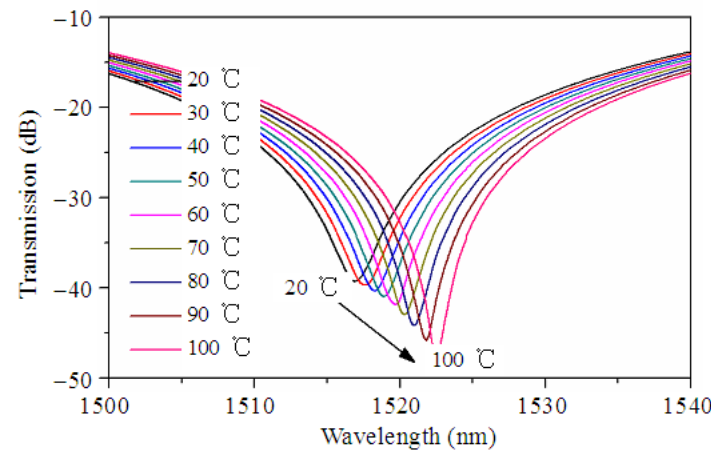

(a)

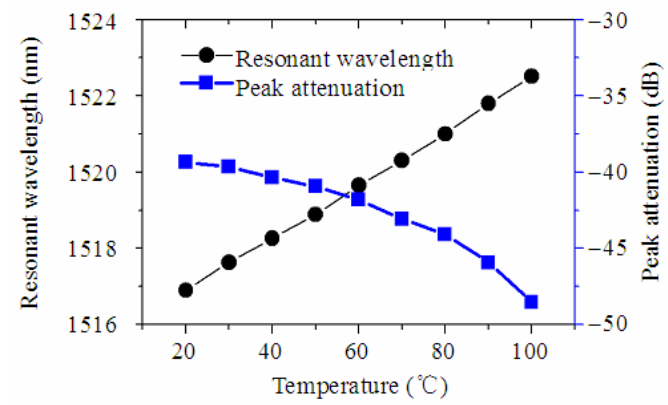

(b)

Fig. 5 Temperature response of the $\mathrm{CO}_{2}$-laser-induced LPFG: (a) transmission spectrum evolution of the $\mathrm{CO}_{2}$-laser-induced LPFG with the rise of temperature and (b) resonant wavelength and peak attenuation of the LPFG as a function of temperature.

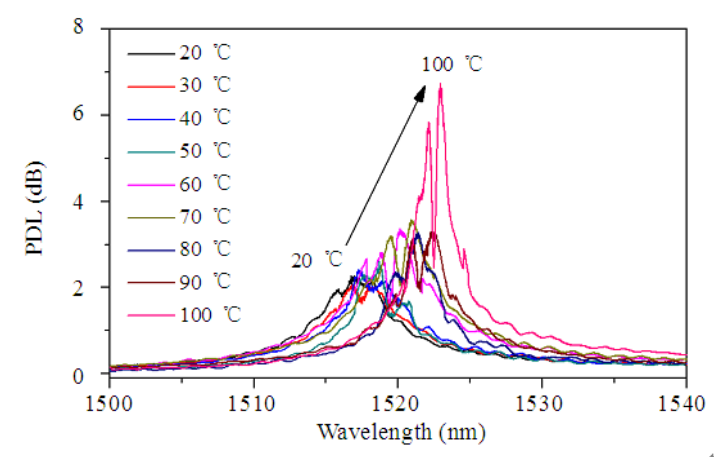

Fig. 6 Polarization dependence evolution of the $\mathrm{CO}_{2}$-laser-induced LPFG with the rise of temperature.

As shown in Fig. 1, periodic grooves are carved on the single-side surface of the fiber. The grooved region of the LPFG is a stress concentration area due to the sharp material deformation. When the temperature rises from the room temperature at which the LPFGs are created, the thermal expansion of the fiber structure will also produce a geometric change in the dimension of the asymmetrical LPFG with periodic grooves. Furthermore, a new stress field is induced in each grooved region due to different thermal strains (or thermal expansion) in the notched and ungrooved sections of the LPFG. As a result, this temperature-induced stress field produces an additional refractive index perturbation in the LPFG due to the photoelastic effect, which increases the refractive index modulation in the LPFG. Moreover, the refractive index of the glass itself varies slightly with temperature (also known as $d n(d T)$, resulting from the well-known thermo-optic effect $[19,20]$. Consequently, the coupling from the fundamental core mode to the cladding mode is enhanced with the rise of temperature, hence significantly enhancing the transmission attenuation of the LPFG, as shown in Fig. 5. In our experiments, we failed to observe the over-coupling between the fundamental mode and the cladding mode in the $\mathrm{CO}_{2}$-laser-induced LPFG due to the limit of temperature adjustment range in the heating oven employed. It is expected that the over-coupling phenomenon occurs if the grating is heated to a higher temperature. In addition, the temperatureinduced strain field is asymmetric within the cross-section of the grooved regions, resulting in asymmetric grooves on the single-side surface of the fiber. As a result, the polarization dependence is also enhanced with the rise of temperature, as shown in Fig. 6.

\section{Discussion}

As discussed above, the mode coupling in the $\mathrm{CO}_{2}$-laser-induced LPFG is very sensitive to the tensile stain applied and the change in external temperature. Hence, after a $\mathrm{CO}_{2}$-laser-induced LPFG is achieved, the coupling efficiency in the grating could be greatly enhanced by means of the two post-treatment techniques: applying a suitable tensile strain to the grating and raising the temperature of the grating. Such post-treatment techniques provide an easy way for achieving a LPFG-based filter with an extremely deep attenuation dip. As is well known, the most important application of LPFGs is to be used as promising band-rejection filters owing to attenuation 
property of LPFGs at desired wavelength. The LPFG-based filters with an extremely deep attenuation dip recently find interesting applications, e.g. subpicosecond pulse-shaping [21]. However, the experimentally attainable level of control, especially for the coupling coefficient, usually does not allow a LPFG to reach the resonant attenuation values over $35 \mathrm{~dB}$ [22]. Fortunately, as shown in Fig. 2, the peak attenuation of the $\mathrm{CO}_{2}$-laser-induced LPFGs can be enhanced to an extremely large value of $-54.82 \mathrm{~dB}$ by applying a suitable tensile strain to obtain the optimum coupling, $k L=\pi / 2$, at the resonant wavelength. In our experiments, an optical spectrum analyzer with a resolution of $0.02 \mathrm{~nm}$ was used to measure the transmission spectrum of the grating. Providing an excellent OSA with a higher wavelength resolution is employed, it is expected to observe a larger peak attenuation of more than $70 \mathrm{~dB}$ at the resonant wavelength while the tensile strain applied is increased by a smaller step.

Moreover, our post-treatment techniques also provide a promising method for tailoring the transmission spectrum of the LPFG to obtain desired attenuation at the expected wavelength after grating fabrication, which is required in another typical application, i.e. gain equalization of erbium-doped fiber amplifiers (EDFAs), of LPFGs. It is usually very difficult, even is impossible, to achieve an LPFG, during grating fabrication, with a desired transmission spectrum that can exactly equalize the gain of an EDFA. As shown in Fig. 2, while a tensile strain is applied to the $\mathrm{CO}_{2}$-laser-induced LPFG, the resonant wavelength shifts toward the longer wavelength and the attenuation is enhanced. Whereas, as shown in Fig. 5, while the temperature of the $\mathrm{CO}_{2}$-laser-induced LPFG rises, the resonant wavelength shifts toward the shorter wavelength, and the attenuation is also enhance. Hence, after an LPFG is written by the $\mathrm{CO}_{2}$-laser-carving technique, its transmission spectrum could be tailored to exactly equalize the gain of an EDFA by the use of the proposed two post-treatment techniques above: applying a suitable tensile strain to the grating and combining with adjusting the temperature of the grating.

\section{Conclusions}

The resonant wavelength of the $\mathrm{CO}_{2}$-laser-induced LPFG with periodic grooves shifts toward the longer wavelength and the attenuation is enhanced with an increase in the tensile strain applied. Moreover, the resonant wavelength of such an LPFG shifts toward the shorter wavelength, and the attenuation is also enhanced with the rise of the external temperature. Based on these unique optical properties of the $\mathrm{CO}_{2}$-laser-induced LPFG, two promising posttreatment techniques, applying tensile strain and raising temperature, are demonstrated to enhance mode-coupling efficiency in the grating. Such two post-treatment techniques can be used to enhance the peak attenuation to achieve an LPFG-based filter with an extremely large attenuation and to tailor the transmission spectrum of the grating to exactly equalize the gain of EDFAs.

\section{Acknowledgement}

This work was supported by the National Natural Science Foundation of China (grant nos. 61425007, 11174064, 61377090, 61575128, and 61308027), Guangdong Provincial Department of Science and Technology (grant nos. 2014A030308007, 2014A030312008, 2014B050504010, and 2015B010105007), Science and Technology Innovation Commission of Shenzhen/Nanshan (grant nos. ZDSYS20140430164957664, KC2014ZDZJ0008A, and GJHZ20150313093755757), and Pearl River Scholar Fellowships.

Open Access This article is distributed under the terms of the Creative Commons Attribution License which permits any use, distribution, and reproduction in any medium, provided the original author(s) and source are credited. 


\section{References:}

[1] D. D. Davis, T. K. Gaylord, E. N. Glytsis, S. G. Kosinski, S. C. Mettler, and A. M. Vengsarkar, "Long-period fibre grating fabrication with focused $\mathrm{CO}_{2}$ laser pulses," Electronics Letters, 1998, 34(3): 302-303.

[2] Y. Rao, Y. Wang, Z. Ran, and T. Zhu, "Novel fiber-optic sensors based on long-period fiber gratings written by high-frequency $\mathrm{CO}_{2}$ laser pulses," Journal of Lightwave Technology, 2003, 21(5): 1320-1327.

[3] Y. Wang and Y. Rao, "Long period fibre grating torsion sensor measuring twist rate and determining twist direction simultaneously," Electronics Letters, 2004, 40(3): 164-166.

[4] Y. Wang, L. Xiao, D. Wang, and W. Jin, "Highly sensitive long-period fiber-grating strain sensor with low temperature sensitivity," Optics Letters, 2006, 31(23): 3414-3416.

[5] Y. Wang, L. Xiao, D. Wang, and W. Jin, "In-fiber polarizer based on a long-period fiber grating written on photonic crystal fiber," Optics Letters, 2007, 32(9): 1035-1037.

[6] Y. Wang, W. Jin, J. Ju, H. Xuan, H. L. Ho, L. Xiao, et al., "Long period gratings in air-core photonic bandgap fibers," Optics Express, 2008, 16(4): 2784-2790.

[7] L. Drozin, P. Y. Fonjallaz, and L. Stensland, "Long-period fibre gratings written by $\mathrm{CO}_{2}$ exposure of $\mathrm{H}_{2}$-loaded, standard fibres," Electronics Letters, 2000, 36(8): 742-744.

[8] Y. Liu and K. S. Chiang, " $\mathrm{CO}_{2}$ laser writing of long-period fiber gratings in optical fibers under tension," Optics Letters, 2008, 33(17): 1933-1935.

[9] H. W. Lee and K. S. Chiang, " $\mathrm{CO}_{2}$ laser writing of long-period fiber grating in photonic crystal fiber under tension," Optics Express, 2009, 17(6): 4533-4539.

[10] R. C. Chaves, A. d. A. P. Pohl, I. Abe, R. Sebem, and A. Paterno, "Strain and temperature characterization of LPGs written by $\mathrm{CO}_{2}$ laser in pure silica LMA photonic crystal fibers," Photonic Sensors, 2015, 5(3): 241-250.

[11] A. M. Vengsarkar, P. J. Lemaire, J. B. Judkins, V. Bhatia, T. Erdogan, and J. E. Sipe, "Long-period fiber gratings as band-rejection filters," Journal of
Lightwave Technology, 1996, 14(1): 58-65.

[12] A. Singh, "Long period fiber grating based refractive index sensor with enhanced sensitivity using Michelson interferometric arrangement," Photonic Sensors, 2014, 5(2): 172-179.

[13] Y. Wang, D. N. Wang, W. Jin, Y. Rao, and G. Peng, "Asymmetric long period fiber gratings fabricated by use of $\mathrm{CO}_{2}$ laser to carve periodic grooves on the optical fiber," Applied Physics Letters, 2006, 89(15): 151105-1-151105-3.

[14] M. Vaziri and C. L. Chen, "Optical-fiber strain sensors with asymmetric etched structures," Applied Optics, 1993, 32(31): 6399-6406.

[15] Y. Wang, J. Chen, and Y. Rao, "Torsion characteristics of long-period fiber gratings induced by high-frequency $\mathrm{CO}_{2}$ laser pulses," Journal of the Optical Society of America B, 2005, 22(6): 1167-1172.

[16] S. Savin, M. J. F. Digonnet, G. S. Kino, and H. J. Shaw, "Tunable mechanically induced long-period fiber gratings," Optics Letters, 2000, 25(10): 710-712.

[17] L. Su, K. S. Chiang, and C. Lu, "Microbend-induced mode coupling in a graded-index multimode fiber," Applied Optics, 2005, 44(34): 7394-7402.

[18] H. Sakata and T. Iwazaki, "Sensitivity-variable fiber optic pressure sensors using microbend fiber gratings," Optics Communications, 2009, 282(23): 4532-4536.

[19] Y. Wang, D. N. Wang, and W. Jin, " $\mathrm{CO}_{2}$ laser-grooved long period fiber grating temperature sensor system based on intensity modulation," Applied Optics, 2006, 45(31): 7966-7970.

[20] Y. Wang, X. Tan, W. Jin, D. Ying, Y. L. Hoo, and S. Liu, "Temperature-controlled transformation in fiber types of fluid-filled photonic crystal fibers and applications," Optics Letters, 2010, 35(1): 88-90.

[21] M. Kulishov and J. Azana, "Long-period fiber gratings as ultrafast optical differentiators," Optics Letters, 2005, 30(20): 2700-2702.

[22] M. Dubov, I. Bennion, S. A. Slattery, and D. N. Nikogosyan, "Strong long-period fiber gratings recorded at $352 \mathrm{~nm}$," Optics Letters, 2005, 30(19): 2533-2535. 